\title{
The Combination of Plasma Fibrinogen Concentration and Neutrophil Lymphocyte Ratio (F-NLR) as a Prognostic Factor of Epithelial Ovarian Cancer
}

This article was published in the following Dove Press journal: OncoTargets and Therapy

Jiani Yang

Jun Ma

\section{Shanshan Cheng}

Yu Wang

Department of Obstetrics and Gynecology, Renji Hospital, School of Medicine, Shanghai Jiaotong University, Shanghai, People's Republic of China
Correspondence: Yu Wang Department of Obstetrics and Gynecology, Renji Hospital, School of Medicine, Shanghai Jiaotong University, Shanghai, People's Republic of China

Tel +86- 2 I 683837I5

Email renjiwangyu@।26.com
Objective: Growing epidemiological evidence supports that coagulation cascades and cancer-associated inflammation are associated with recurrence and survival of epithelial ovarian cancer (EOC). This study aimed to assess the clinical significance of the combination of plasm fibrinogen and neutrophil lymphocyte ratio (F-NLR) score to predict EOC prognosis, including recurrence, disease-free survival (DFS), and overall survival (OS).

Patients and Methods: We retrospectively enrolled 281 EOC patients who underwent surgery at our institution. According to receiver operating characteristic curve, cut-off values of fibrinogen and NLR were set at $3.44 \mathrm{~g} / \mathrm{L}$ and 2.46 , respectively, to predict recurrence. The F-NLR score was then classified into three groups as follows: F-NLR score of 2: both hyperfibrinogenemia $(>3.44 \mathrm{mg} / \mathrm{dL})$ and high NLR $(>2.46)$, F-NLR score of 1: either hyperfibrinogenemia or high NLR, and F-NLR score of 0 : neither of the abnormalities. Continuous and categorical variables were compared using $T$-test and chi-square test among F-NLR groups. The Cox hazard regression model was used to assess prognostic factors. Both DFS and OS curves were generated by Kaplan-Meier method.

Results: The analyses showed that F-NLR was significantly associated with clinical stage $(P=0.000)$, lymphatic metastasis $(P=0.001)$, and carbohydrate antigen (CA) 125 level $(P=0.048)$. The F-NLR (hazard ratio $=2.211 ; 95 \%$ confidence interval $=1.251-6.394 ; P=0.002$ ) was demonstrated as an independent prognostic factor for survival of EOC. The DFS rates in F-NLR groups 0,1 , and 2 were $68.5 \%, 47.7 \%$, and $31.7 \%$, respectively $(P=0.000)$; the OS rates in previous groups were $75.9 \%, 51.4 \%$, and $34.2 \%(P=0.000)$ and the 5 -year survival times (mean \pm SD, months) were $64.24 \pm 24.21,60.27 \pm 22.65$, and $46.09 \pm 20.32$, respectively. By subgroup analysis, the F-NLR was significantly associated with DFS and OS among patients with advanced tumor stage (Stage III or IV) or lymphatic metastasis.

Conclusion: The pre-operative F-NLR score, a novel inflammation-based grading system, was a promising prognosis predictor for EOC patients, especially those with advanced clinical stage and those with lymph node metastasis.

Keywords: fibrinogen, neutrophil-lymphocyte ratio, epithelial ovarian cancer, prognosis, F-NLR

\section{Introduction}

Ovarian cancer is the fifth cause of cancer-related death among women and the leading cause of death among gynecological cancers, with 13,940 deaths and 21,750 new cases estimated for 2020 in the US. ${ }^{1}$ More than $70 \%$ of ovarian cancers 
are diagnosed in advanced stages, partly due to the lack of early symptoms and physical signs. ${ }^{2}$ Despite development in treatment and diagnosis over the past decades, the prognosis for advanced stages is still poor, with a 10year survival rate of $5-21 \% .^{3}$ Moreover, approximately $80 \%$ of patients diagnosed with EOC will relapse after initial therapy of standard surgery followed by platinumpaclitaxel based chemotherapy. ${ }^{4}$ Given the poor prognosis, effective and easy-obtained methods for accurately predicting progression, metastasis, and prognosis of EOC patients are urgently required to improve patient survival. ${ }^{5}$

The CA-125 and Human epididymis protein 4 (HE4), as a routinely-used tumor-specific antigen, has low sensitivity $(50-62 \%)$ and limited specificity $(73-77 \%)$ to predict ovarian cancer progression. ${ }^{6,8}$ Therefore, new methods for predicting the prognosis of epithelial ovarian cancer (EOC) patients after surgical resection is of clinical significance to improve survival. ${ }^{7}$

Systemic inflammatory response plays a pivotal role in the cancer progression, by promoting proliferation of tumor cells and accelerating metastasis. ${ }^{9}$ The neutrophilto-lymphocyte ratio (NLR), as a representative index of inflammatory status, has been reported as a prognostic marker for many solid malignancies, including ovarian cancer. ${ }^{10,11}$ Neutrophils promote cancer progression by producing adequate cytokines and chemokines that could provide the tumor microenvironment. ${ }^{12}$ In terms of tumorrelated inflammatory, the coagulation cascade also plays an important role in the malignant process of cancer progression and metastasis. ${ }^{13}$ Fibrinogen, as a key factor of coagulation cascade, is a liver-produced pro-inflammatory protein transformed from fibrin through activated thrombin. ${ }^{14}$ Recent studies demonstrated that hyperfibrinogenemia could promote cancer aggressiveness and relate to poor prognosis in various malignancies. ${ }^{10,15}$ To date, the combination of fibrinogen and NLR (F-NLR) has been focused on as a novel prognostic index in several types of malignancies, including gastric cancer, lung cancer, and esophageal squamous cell carcinoma. ${ }^{16,17}$ However, there have been no current studies regarding a combined analysis based on plasma F-NLR as a predictor of tumor response and prognosis in ovarian cancer. This study aimed to evaluate the prognostic value of F-NLR, a combined score based on cut-off values of fibrinogen concentration and NLR among EOC patients. Furthermore, the present study also assessed the correlation between three F-NLR groups and clinicopathologic features or clinical laboratory variables of EOC patients to predict its potential clinical application.

\section{Patients and Methods Patients}

We retrospectively reviewed data from 337 patients with pathologically diagnosed EOC who underwent standardized surgery at the Department of Obstetrics and Gynecology, Renji Hospital Affiliated to Shanghai Jiaotong University School of Medicine between May 2008 and February 2015. The criteria for inclusion in this study included: 1) histologically confirmed EOC; 2) no coexisting cancers or prior cancers within 5 years; 3) with complete follow-up data; and 4) underwent standardized surgery aimed to achieve optimal tumor debulking. Patients were excluded from our research if they: 1) were lost to follow-up ( $n=19) ; 2)$ were without detailed post-therapeutic clinical, laboratory, imaging data $(\mathrm{n}=15)$; 3) underwent preoperative treatments, such as neoadjuvant therapy or radiotherapy $(\mathrm{n}=6)$; and 4$)$ had clinical evidence of infection, hematological disorder, or autoimmune disease that might result in hyperfibrinogenemia or high NLR $(\mathrm{n}=16)$. Finally, 281 patients were assessed in the analysis (Figure 1). The power for the endpoint cancer recurrence and overall survival is calculated based on a two sided $t$-test with a significance level of $5 \%$. With the final sample size of 281 subjects, the trial have more than $80 \%$ power to detect a difference of cancer recurrence between each F-NLR groups.

The clinical stage of EOC was assessed and referred to the International Federation of Gynecology and Obstetrics (FIGO) stage. The routine blood tests, routine coagulation tests, and tumor marker measurements, including CA-125, CA-199, HE4, Alpha Fetoprotein (AFP), and Carcinoembryonic Antigen (CEA), were conducted within 3 days before surgery. The clinicopathologic variables, including age, Body Mass Index (BMI), menopausal status, fertility history, comorbidities, tumor size, FIGO clinical stage, histologic grade, lymph node metastasis, postoperative complications, and data for routine laboratory measurements were collected retrospectively from medical records. The operation was aimed at maximal ovarian tumor resection without visible residual tumor to achieve optimal tumor debulking, of which the size of the residual tumor was less than $1 \mathrm{~cm}$ at the longest diameter. The operation was followed by standardized platinumbased chemotherapy. Overall survival (OS) was identified from the date of operation to last follow-up visit or death. 


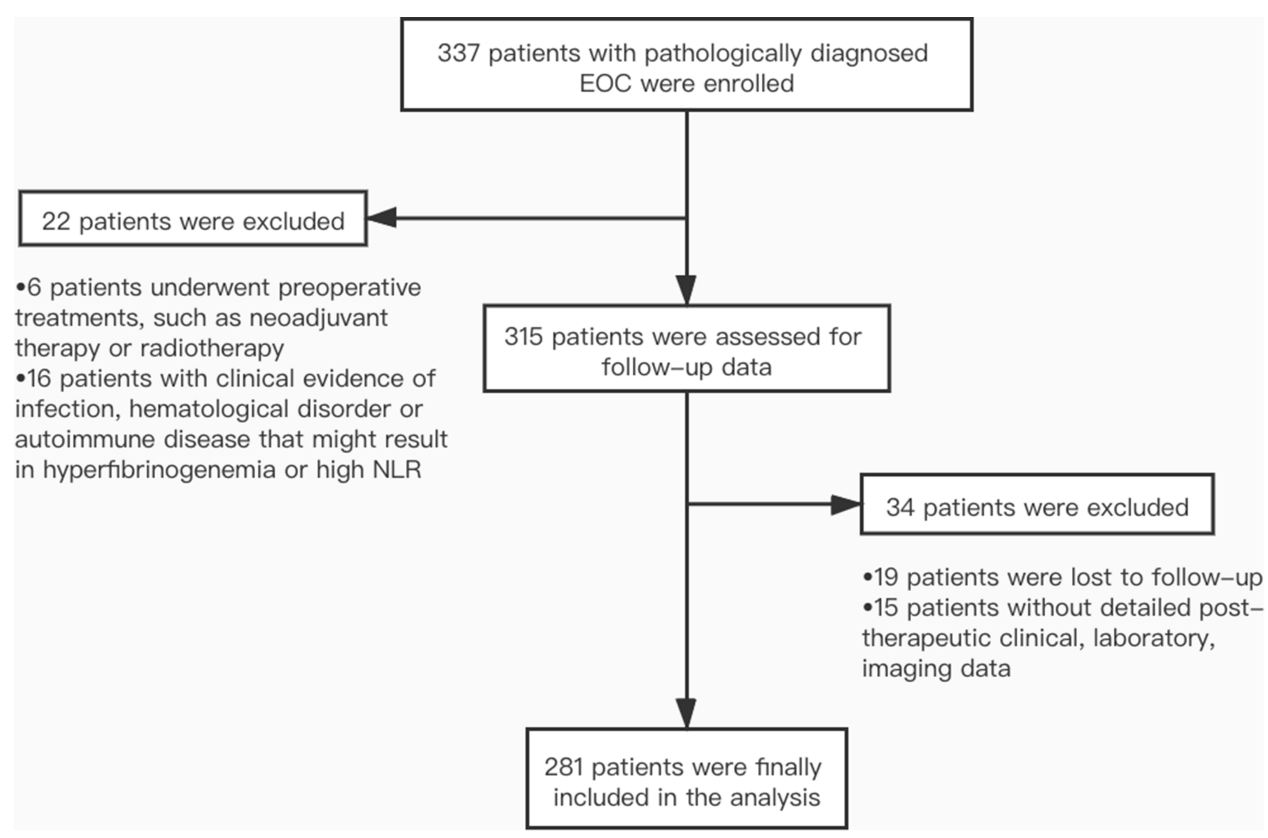

Figure I Patient enrollment flow chart of the study.

Disease-free survival (DFS) was measured from the date of operation to last follow-up visit or relapse, which was identified by the latest radiographic evidence. Ethical approval was obtained from the Ethics Committee of Renji Hospital Affiliated to Shanghai Jiaotong University School of Medicine and all patients provided informed consent for the use of their information.

\section{F-NLR Measurement}

Routine laboratory measurements including serum levels of fibrinogen, neutrophil, and lymphocyte were extracted retrospectively from the medical records of patients involved. Blood tests were administered within 3 days prior to surgery. For those patients who had multiple blood tests within 3 days before operation, the average of the multiple test data was used for analysis. The NLR was defined as the neutrophil count divided by lymphocyte count. According to the Youden index by Receiver operating characteristic (ROC) curve to predict cancer recurrence, the cut-off value was set at 2.46 for NLR and $3.44 \mathrm{~g} / \mathrm{L}$ for fibrinogen concentrations (Figure 2). For these values, sensitivity was determined as $70.54 \%$ and $86.61 \%$, while specificity was determined as $60.61 \%$ and $69.70 \%$, respectively. Based on the ROC curve, the area under concentration-time curve (AUC) was $0.675(P=0.00$, 95\% CI $=0.608-0.742)$ for NLR and $0.747(P=0.00,95 \%$ $\mathrm{CI}=0.681-0.812)$ for fibrinogen. The F-NLR score was then classified into three groups referring to each cut-off value of plasma fibrinogen level and NLR as follows: an F-NLR score

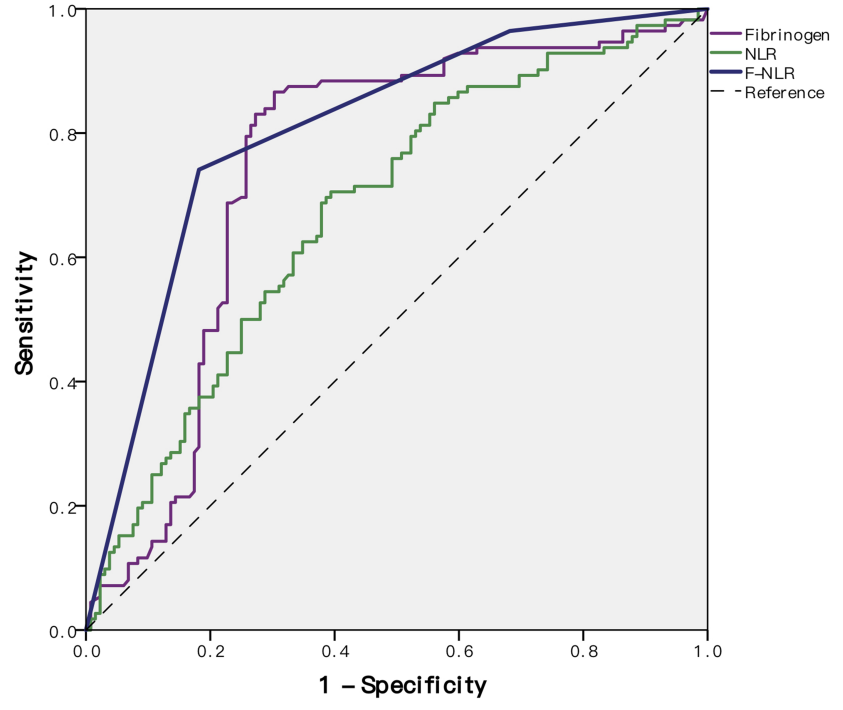

Figure 2 Receiver operating characteristic (ROC) curves to assess the predictive value of fibrinogen concentrations and neutrophil lymphocyte ratio (F-NLR).

of 2: both hyperfibrinogenemia ( $>3.44 \mathrm{mg} / \mathrm{dL})$ and high NLR $(>2.46)$, an F-NLR score of 1: either hyperfibrinogenemia $(>3.44 \mathrm{mg} / \mathrm{dL})$ or high NLR $(>2.46)$, and an F-NLR score of 0 : with neither of these hematological abnormalities. For the F-NLR score, the AUC value was $0.806(P=0.00,95 \%$ $\mathrm{CI}=0.751-0.862)$ referring to the ROC curve.

\section{Statistical Methods}

The $T$-test and chi-square test were applied to evaluate the differences of clinicopathologic characteristics or clinical 
laboratory variables between groups for continuous and categorical variables, respectively. ROC curve analysis was used to identify the cut-off value of fibrinogen concentrations and NLR according to the maximal Youden index. Prognostic factors were determined using both univariate and multivariate analyses through the Cox's proportional hazards regression model. Kaplan-Meier methods were used to generate the survival curves. The prognostic differences were assessed by Log rank test. All statistical analyses were conducted through SPSS statistical software version 23 (IBM) and graphed using Graph Prism Version 7.0a (GraphPad Software). The $P$-value $<0.05$ was defined as statistically significant.

\section{Results}

\section{Clinical Baseline Characteristics of EOC Patients}

The overall demographic and clinical features of all the EOC patients involved are summarized in Tables 1 and 2, grouped by the F-NLR score. A total of 281 pathologically diagnosed EOC patients who met the inclusion criteria were finally included in the analysis, with the mean age of 57.58 \pm 11.88 years old. Patients with clinical stages I, II, III, and IV accounted for 23 (8.19\%), 51 (18.15\%), 185 (65.84\%), and $22(7.83 \%)$, respectively (Figure 3A). There were $180(64.1 \%)$ patients with histology-proved lymphatic

Table I The Correlation Between Preoperative F-NLR Score and Clinicopathological Features of the Epithelial Ovarian Cancer (EOC) Patients Included

\begin{tabular}{|c|c|c|c|c|c|}
\hline & \multirow[t]{2}{*}{ All Patients $(n=28 I)$} & \multicolumn{3}{|l|}{ F-NLR Score } & \multirow[t]{2}{*}{$P$-value } \\
\hline & & F-NLR=0 $(n=54)$ & $F-N L R=I \quad(n=\mid 07)$ & F-NLR=2 $(n=\mid 20)$ & \\
\hline Age (years) & $57.58 \pm \mid 1.88$ & $58.39 \pm 11.89$ & $57.50 \pm 10.85$ & $57.83 \pm 11.03$ & 0.118 \\
\hline BMI $\left(\mathrm{kg} / \mathrm{m}^{2}\right)$ & $22.46 \pm 0.82$ & $21.98 \pm 0.81$ & $22.48 \pm 0.64$ & $22.98 \pm 0.64$ & 0.133 \\
\hline Menopausal status, n (\%) & & & & & 0.219 \\
\hline Pre/peri-menopause & 97 (34.5\%) & $15(5.3 \%)$ & $34(12.1 \%)$ & 48 (I7.1\%) & - \\
\hline Post-menopause & I 84 (65.5\%) & 39 (I3.9\%) & $73(26.0 \%)$ & $72(25.6 \%)$ & - \\
\hline Fertility history, n (\%) & & & & & 0.521 \\
\hline 0 & $13(4.6 \%)$ & $3(1.1 \%)$ & $4(1.4 \%)$ & $6(2.1 \%)$ & - \\
\hline I & I29 (45.9\%) & 27 (9.6\%) & 54 (19.2\%) & 48 (I7.1\%) & - \\
\hline$\geq 2$ & $139(49.5 \%)$ & 24 (8.5\%) & 49 (I7.4\%) & $66(23.5 \%)$ & - \\
\hline Comorbidities, n (\%) & & & & & - \\
\hline Cardiovascular disease & 21 (7.5\%) & $7(2.5 \%)$ & $6(2.1 \%)$ & $8(2.8 \%)$ & 0.223 \\
\hline Diabetes & $14(5.0 \%)$ & $5(1.8 \%)$ & $4(1.4 \%)$ & $5(1.8 \%)$ & 0.272 \\
\hline Hypertension & $22(7.8 \%)$ & 7 (2.5\%) & 7 (2.5\%) & $8(2.8 \%)$ & 0.295 \\
\hline Tumor size $(\mathrm{cm})$ & $7.31 \pm 4.29$ & $8.10 \pm 5.62$ & $7.09 \pm 5.06$ & $7.29 \pm 4.75$ & 0.119 \\
\hline Pathological grade, n (\%) & & & & & 0.486 \\
\hline GI-2 & $80(28.5 \%)$ & $18(6.4 \%)$ & $32(\mathrm{II} .4 \%)$ & 30 (10.7\%) & - \\
\hline G3 & 201 (7I.5\%) & $36(12.8 \%)$ & 75 (26.7\%) & $90(32.0 \%)$ & - \\
\hline Clinical stage, n (\%) & & & & & 0.000 \\
\hline I-II & $74(26.3 \%)$ & 21 (7.5\%) & $36(12.8 \%)$ & $17(6.0 \%)$ & - \\
\hline III-IV & 207 (73.7\%) & $33(11.7 \%)$ & 71 (25.3\%) & $103(36.7 \%)$ & - \\
\hline Histological type, n (\%) & & & & & 0.640 \\
\hline Serous & $186(66.2 \%)$ & 31 (1I.0\%) & $69(24.6 \%)$ & $86(30.6 \%)$ & - \\
\hline Mucinous & $33(11.7 \%)$ & $8(2.8 \%)$ & $12(4.3 \%)$ & $13(4.6 \%)$ & - \\
\hline Endometrioid & $24(8.5 \%)$ & $6(2.1 \%)$ & $9(3.2 \%)$ & 9 (3.2\%) & - \\
\hline Others & 38 (I3.5\%) & 9 (3.2\%) & 17 (6.0\%) & $12(4.3 \%)$ & - \\
\hline Lymphatic metastasis, $\mathrm{n}(\%)$ & & & & & 0.001 \\
\hline Negative (-) & I0I (35.9\%) & $28(10.0 \%)$ & 48 (17.1\%) & 25 (8.9\%) & - \\
\hline Positive (+) & $180(64.1 \%)$ & $26(9.3 \%)$ & 59 (21.0\%) & 95 (33.8\%) & - \\
\hline
\end{tabular}

Abbreviations: BMI, body mass index; F-NLR, fibrinogen and neutrophil-lymphocyte ratio score. 
Table 2 The Correlation Between Preoperative F-NLR Score and Clinical Laboratory Characteristics of the Epithelial Ovarian Cancer (EOC) Patients Included

\begin{tabular}{|c|c|c|c|c|c|}
\hline & \multirow[t]{2}{*}{ All Patients $(n=28 I)$} & \multicolumn{3}{|l|}{ F-NLR Score } & \multirow[t]{2}{*}{$P$-value } \\
\hline & & F-NLR=0 $(n=54)$ & F-NLR $=I \quad(n=107)$ & F-NLR=2 $(n=\mid 20)$ & \\
\hline Neutrophil (10^9/L) & $5.09 \pm 2.63$ & $3.59 \pm 1.08$ & $4.84 \pm 2.83$ & $6.05 \pm 2.62$ & 0.000 \\
\hline Lymphocyte (I0^9/L) & $1.39 \pm 0.55$ & $1.78 \pm 0.55$ & $1.38 \pm 0.56$ & $1.23 \pm 0.43$ & 0.000 \\
\hline Platelet (I0^9/L) & $271.61 \pm 105.58$ & $265.85 \pm 60.60$ & $272.43 \pm 103.47$ & $278 \pm 110.50$ & 0.074 \\
\hline Albumin $(g / L)$ & $38.86 \pm 6.04$ & $38.12 \pm 5.60$ & $39.34 \pm 5.15$ & $38.03 \pm 6.42$ & 0.372 \\
\hline Fibrinogen (g/L) & $3.75 \pm 2.09$ & $3.22 \pm 1.96$ & $4.12 \pm 2.98$ & $5.47 \pm 3.12$ & 0.000 \\
\hline CA-I25 (U/mL) & $1055 \pm 504.85$ & $839.14 \pm 446.17$ & $1002.75 \pm 817.10$ & $1130.73 \pm 687.78$ & 0.048 \\
\hline CA-I99 (U/mL) & $82.72 \pm 65.4 I$ & $79.23 \pm 77.48$ & $85.99 \pm 53.48$ & $83.75 \pm 70.61$ & 0.569 \\
\hline $\operatorname{AFP}(\mathrm{ng} / \mathrm{mL})$ & $4.90 \pm 4.41$ & $4.72 \pm 3.07$ & $4.89 \pm 4.85$ & $5.03 \pm 1.86$ & 0.439 \\
\hline CEA $(\mathrm{ng} / \mathrm{mL})$ & $2.93 \pm 2.31$ & $3.38 \pm 2.60$ & $2.89 \pm 2.77$ & $2.97 \pm 2.86$ & 0.897 \\
\hline HE4 (pmol/l) & $584.24 \pm 426.65$ & $522.92 \pm 443.57$ & $578.48 \pm 486.52$ & $655.16 \pm 561.99$ & 0.926 \\
\hline
\end{tabular}

Abbreviations: AFP, alpha fetoprotein; CA-125, carbohydrate antigen 125; CA-199, carbohydrate antigen 199; CEA, carcinoembryonic antigen; F-NLR, fibrinogen and neutrophil-lymphocyte ratio score; HE4, human epididymis protein 4.

metastasis, and $80(64.1 \%)$ patients presented with low pathological grade (G1 and G2) (Figure 3B and C). The F-NLR score of all patients was allocated as follows: $\mathrm{F}-\mathrm{NLR}=0,54$ (19.2\%) patients; F-NLR=1, 107 (38.1\%) patients; and F-NLR=2, $120(42.7 .7 \%)$ patients. The 5 -year OS rate and DFS rate in the entire study population was $47.7 \%$ and $41.3 \%$, respectively. The median follow-up for patients was 60 months (range $=44-79$ months). Only five patients reported postoperative complications, including bleeding $(n=1)$, intestinal obstruction $(n=2)$, and thrombosis $(n=2)$. All the patients involved received surgery followed by standardized platinum-based chemotherapy, while 17 of the 281 patients also received post-operation radiation therapy.
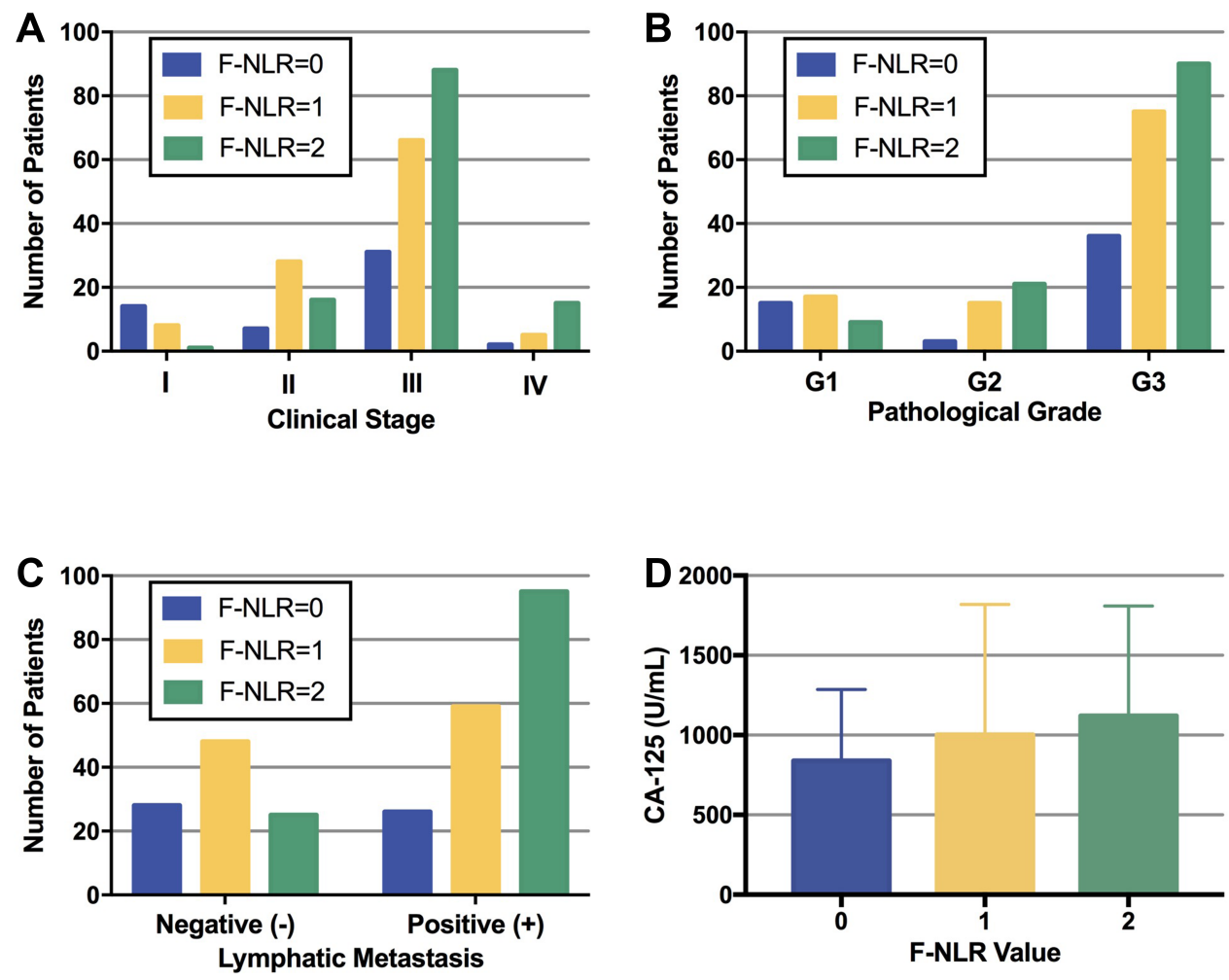

Figure 3 The fibrinogen concentrations and neutrophil lymphocyte ratio (F- NLR) score in patients with epithelial ovarian cancer grouped by (A) the International Federation of Gynecology and Obstetrics (FIGO) clinical stage, (B) pathological grade, (C) lymphatic metastasis, and (D) CA-I25 level. 


\section{Correlation Analysis Between F-NLR Score and Clinicopathological Factors in EOC Patients}

The association between the preoperative F-NLR score and clinicopathologic characteristics of EOC patients was also shown in Tables 1 and 2. We found significant correlation of F-NLR with clinical stage $(P=0.000)$, lymphatic metastasis $(P=0.001)$, neutrophil count $(P=0.000)$, lymphocyte count $(P=0.000)$, fibrinogen concentration $(P=0.000)$, and CA-125 $(P=0.048)$ (Figure 3$)$. The mean CA-125 $( \pm \mathrm{SD})$ values in patients with various F-NLR scores of 0,1 , and 2 were $839.14 \pm 446.17,1002.75$ \pm 817.10 , and $1130.73 \pm 687.78 \quad(\mathrm{U} / \mathrm{mL})$, respectively (Figure 3D). No significant differences among three F-NLR groups were found for age, BMI index, menopausal status, fertility history, comorbidities, tumor size, pathological tumor grade, histological type, platelet, albumin, CA-199, AFP, CEA, and HE4 ( $P \geq 0.05)$.

\section{Univariate and Multivariate Regression Analyses for OS}

To further determine the independent predictive indexes, both univariate and multivariate analyses including the variables mentioned are provided in Table 3. The clinical stage $(\mathrm{HR}=1.623 ; 95 \% \mathrm{CI}=1.171-2.712 ; P=0.035)$, lymph node metastasis $\quad(\mathrm{HR}=2.034 ; 95 \% \mathrm{CI}=1.208-3.425 ; P=0.008)$, F-NLR score $(\mathrm{HR}=2.991 ; 95 \% \mathrm{CI}=1.668-6.149 ; P=0.000)$, and $\mathrm{CA}-125(\mathrm{HR}=1.249 ; 95 \% \mathrm{CI}=1.184-1.426 ; P=0.025)$ were significantly associated with OS in the univariate analyses. Then, these indicators were included in the multivariate Cox hazards model. The analyses demonstrated that the clinical stage $(\mathrm{HR}=1.829 ; 95 \% \mathrm{CI}=1.055-3.736 ; P=0.042)$, lymph node metastasis $(\mathrm{HR}=2.153 ; 95 \% \mathrm{CI}=1.037-4.532$; $P=0.033)$, F-NLR score $(\mathrm{HR}=2.211 ; 95 \% \mathrm{CI}=1.251-6.394$; $P=0.002)$, and $\mathrm{CA}-125$ (HR=1.316; 95\% $\mathrm{CI}=1.012-1.729$; $P=0.023)$ were independent prognostic factors for OS of EOC patients (Table 3).

\section{F-NLR as Prognostic Factors for EOC Patients}

For all EOC patients, the DFS rates among F-NLR groups 0, 1 , and 2 were $68.5 \%$ (37/54), $47.7 \%$ (51/107), and $31.7 \%$ (38/120), respectively $(P<0.0001$, Figure $4 \mathrm{~A})$. The OS rates in F-NLR groups 0,1 , and 2 also differed significantly and were $75.9 \%$ (41/54), 51.4\% (55/107), and 34.2\% (41/120), respectively $(P<0.0001$, Figure $4 \mathrm{~B})$. Moreover, subgroup analysis presented that F-NLR score was significantly associated with both DFS and OS in EOC patients with clinical stage III-IV $(P<0.0001)$ and lymph node metastasis $(P<0.0001)$ according to the Log-rank test. For stage III and IV EOC patients, the DFS rates among F-NLR groups 0,1 , and 2 were $66.7 \%(22 / 33), 46.4 \%(33 / 71)$, and $26.2 \%$ (27/103), respectively. The OS rates in this subgroup differed significantly among F-NLR groups and were $69.7 \%$ (23/33), 52.1\% (37/71), and 34.0\% (35/103), respectively. For lymph node metastasis positive $(+)$ EOC patients, the DFS rates among F-NLR groups 0,1 , and 2 were $50.0 \%$ (13/26), 42.4\% (25/59), and 27.4\% (26/95), respectively. The OS rates in these patients differed significantly among F-NLR groups and were $57.7 \%$ (15/26), 47.5\% (28/59), and $32.6 \%$ (31/95), respectively. Results are shown in Figures 5 and 6. Higher F-NLR score was significantly related to poorer prognosis, especially among EOC patients with advanced clinical stage (Stage III and IV) and those with lymphatic metastasis.

\section{Discussion}

In this study, we analyzed the combination of fibrinogen and NLR together as F-NLR to investigate its clinical significance for prognosis of EOC patients. To the best of our knowledge, although the clinical value of F-NLR as a prognostic marker was demonstrated in various malignancies, this is the first study to further assess its application in EOC.

Increasing evidence has indicated that systematic inflammation responses and immune cells, the basic components of a tumor microenvironment, could be crucial prognostic indicators for malignancies. ${ }^{18}$ Fibrinogen, a liver-produced protein transformed from fibrin by activated thrombin, would increase when a malignant neoplasm or systemic inflammation is present. ${ }^{19}$ Many studies have investigated the causes of hyperfibrinogenemia in malignant tumors, though further studies are still needed to elucidate the detailed underlying biomechanism. ${ }^{20}$ Sahni et $\mathrm{al}^{21}$ indicated that tumor cells could exert interleukin- 6 to accelerate the transformation of fibrinogen in patients. Through the interaction with vascular endothelial growth factor (VEGF) and fibroblast growth factor-2 (FGF-2), fibrinogen could stimulate tumor proliferation and new vessel formation. ${ }^{21}$ Our study assessed the clinical value of plasma fibrinogen concentrations to predict ovarian cancer recurrence with the AUC of $0.747(P=0.00,95 \% \mathrm{CI}=0.681-0.812)$ based on the ROC curve. 
Table 3 Univariate and Multivariate Regression Analyses of Clinical Characteristics in Relation to Overall Survival (OS) of Epithelial Ovarian Cancer (EOC) Patients

\begin{tabular}{|c|c|c|c|c|c|c|}
\hline & \multicolumn{3}{|c|}{ Univariate Analysis } & \multicolumn{3}{|c|}{ Multivariate Analysis } \\
\hline & Hazard Ratio & $95 \% \mathrm{Cl}$ & $P$-value & Hazard Ratio & $95 \% \mathrm{Cl}$ & $P$-value \\
\hline Age (years) & 1.003 & $0.985-1.021$ & 0.767 & 0.972 & $0.916-1.052$ & 0.833 \\
\hline BMI $\left(\mathrm{kg} / \mathrm{m}^{2}\right)$ & 0.929 & $0.718-1.202$ & 0.575 & 0.850 & $0.735-1.186$ & 0.759 \\
\hline Tumor size $(\mathrm{cm})$ & 0.970 & $0.928-1.013$ & 0.171 & 0.978 & $0.913-1.061$ & 0.557 \\
\hline \multicolumn{7}{|l|}{ Menopausal status } \\
\hline Pre/peri-menopause & Reference & - & - & Reference & - & - \\
\hline Post-menopause & 0.826 & $0.491-1.218$ & 0.283 & 0.938 & $0.714-1.438$ & 0.324 \\
\hline \multicolumn{7}{|l|}{ Fertility history } \\
\hline 0 & Reference & - & - & Reference & - & - \\
\hline I & 0.912 & $0.820-1.147$ & 0.493 & 0.973 & $0.902-1.157$ & 0.516 \\
\hline$\geq 2$ & 1.028 & $0.728-1.342$ & 0.521 & 1.227 & $0.924-1.735$ & 0.574 \\
\hline \multicolumn{7}{|l|}{ Comorbidities } \\
\hline Cardiovascular disease & 0.825 & $0.698-1.120$ & 0.288 & 0.954 & $0.814-1.367$ & 0.331 \\
\hline Diabetes & 1.099 & $0.830-1.158$ & 0.431 & 1.063 & $0.789-1.282$ & 0.443 \\
\hline Hypertension & 1.025 & $0.817-1.239$ & 0.873 & 1.097 & $0.942-1.158$ & 0.892 \\
\hline \multicolumn{7}{|l|}{ Pathological grade } \\
\hline GI-2 & Reference & - & - & Reference & - & - \\
\hline G3 & 0.758 & $0.444-1.293$ & 0.309 & 0.784 & $0.352-|.73|$ & 0.582 \\
\hline \multicolumn{7}{|l|}{ Clinical stage } \\
\hline I-II & Reference & - & - & Reference & - & - \\
\hline III-IV & 1.623 & $1.17 \mid-2.712$ & 0.035 & 1.829 & $1.055-3.736$ & 0.042 \\
\hline \multicolumn{7}{|l|}{ Lymphatic metastasis } \\
\hline Negative (-) & Reference & - & - & Reference & - & - \\
\hline Positive (+) & 2.034 & $1.208-3.425$ & 0.008 & 2.153 & $1.037-4.532$ & 0.033 \\
\hline Platelet (I0^9/L) & 1.002 & $1.000-1.004$ & 0.067 & 0.998 & $0.995-1.001$ & 0.097 \\
\hline Albumin (g/L) & 0.963 & $0.931-1.007$ & 0.133 & 0.978 & $0.905-1.014$ & 0.237 \\
\hline F-NLR score & & & 0.000 & & & 0.002 \\
\hline$F-N L R=0$ & Reference & - & - & Reference & - & - \\
\hline $\mathrm{F}-\mathrm{NLR}=\mathrm{I}$ & 1.337 & $1.012-4.854$ & 0.012 & 2.891 & $1.809-5.124$ & 0.043 \\
\hline $\mathrm{F}-\mathrm{NLR}=2$ & 2.991 & $1.668-6.149$ & 0.000 & 2.211 & $1.25 \mid-6.394$ & 0.002 \\
\hline CA-I25 (U/mL) & 1.249 & I.184-I.426 & 0.025 & 1.316 & $1.012-1.729$ & 0.023 \\
\hline CA-I99 (U/mL) & 0.973 & $0.918-1.046$ & 0.851 & 0.971 & $0.931-1.073$ & 0.327 \\
\hline $\operatorname{AFP}(\mathrm{ng} / \mathrm{mL})$ & 1.000 & $0.991-1.008$ & 0.917 & 0.875 & $0.623-|.27|$ & 0.122 \\
\hline CEA $(\mathrm{ng} / \mathrm{mL})$ & 0.995 & $0.958-1.034$ & 0.798 & 0.980 & $0.932-1.050$ & 0.571 \\
\hline HE4 (pmol/l) & 1.301 & $0.978-1.830$ & 0.522 & 1.934 & $0.97 I-2.224$ & 0.832 \\
\hline
\end{tabular}

Abbreviations: AFP, alpha fetoprotein; BMI, body mass index; CA-125, carbohydrate antigen 125; CA-199, carbohydrate antigen 199; CEA, carcinoembryonic antigen; Cl, confidence interval; F-NLR, fibrinogen and neutrophil-lymphocyte ratio score; HE4, human epididymis protein 4.

Lymphocytes play important roles in immunesurveillance and the inhibition of tumor cell proliferation and migration. ${ }^{22}$ Many types of lymphocytes, such as T-cells, monocytes, and macrophages, perform important functions to infiltrate EOC. ${ }^{23}$ For instance, studies showed that T-cells could exert a killing effect towards tumor cells and induce target cell apoptosis in patients with ovarian cancer. ${ }^{24}$ Since lymphocytes serve as key components of anti-cancer immunity, decreased lymphocyte counts in blood could lead to a downregulation of immune response against cancer. Likewise, circulating neutrophils are also revealed as vital components of tumor inflammation responses. ${ }^{23}$ Kusumanto et $\mathrm{al}^{25}$ demonstrated that neutrophils could produce various cytokines, including VEGF, 

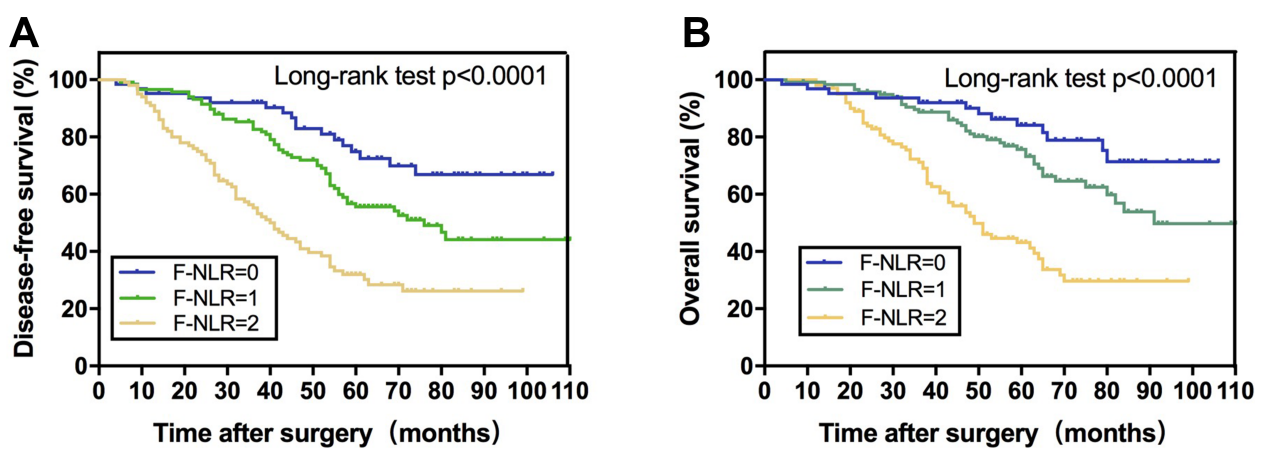

Figure 4 Kaplan-Meier survival curves of all epithelial ovarian cancer patients stratified by fibrinogen concentrations and neutrophil lymphocyte ratio (F-NLR) score for (A) disease-free survival (DFS) and (B) overall survival (OS).

A

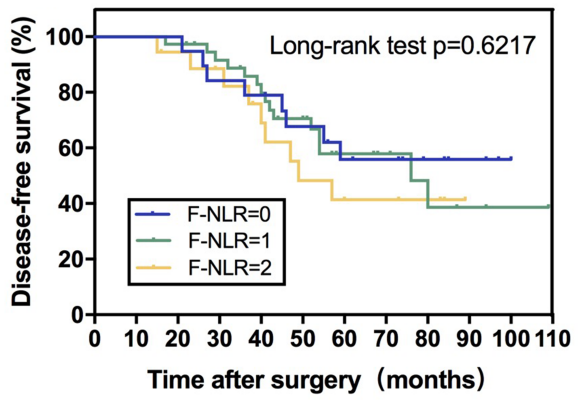

C

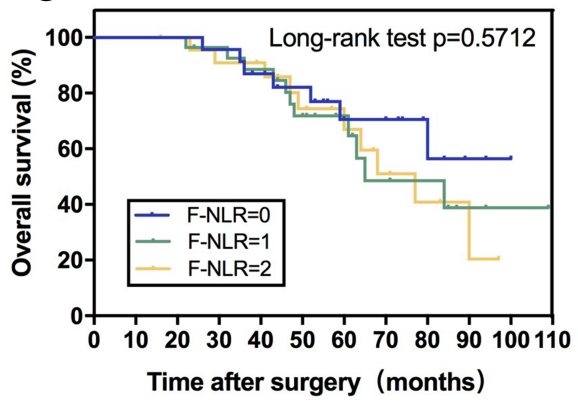

B

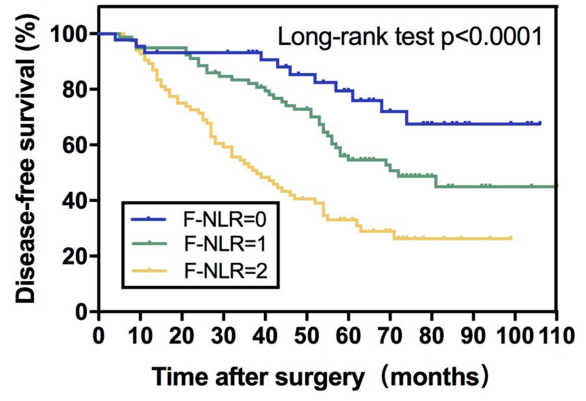

D

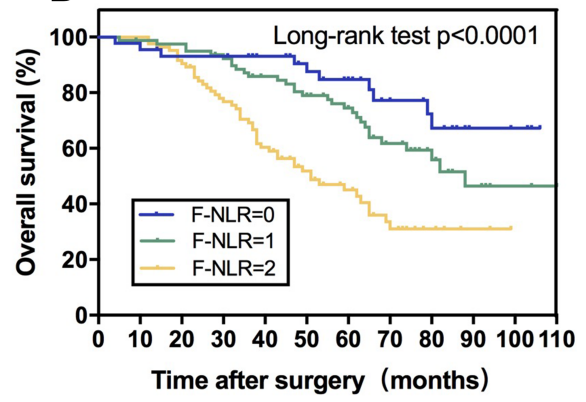

Figure 5 Kaplan-Meier survival curves stratified by fibrinogen concentrations and neutrophil lymphocyte ratio (F-NLR) score among patients with (A) clinical stage I and II for disease-free survival (DFS), (B) clinical stage III and IV for DFS; (C) clinical stage I and II for overall survival (OS), and (D) clinical stage III and IV for OS.

tumor necrosis factor- $\alpha$, and interleukin, which contribute to tumor metastasis. To date, NLR, the combination of neutrophil and lymphocyte counts, could be used as a crucial index to indicate the systemic inflammatory response in various cancers. ${ }^{26}$ In our study, we also found that the NLR before operation could be a significant predictor for recurrence in ovarian cancer patients $(P=0.00,95 \% \mathrm{CI}=0.608-0.742)$.

Based on these previous studies, both fibrinogen and NLR are potential predictive markers for cancer prognosis. Hence, we simultaneously investigated whether the combination of plasma fibrinogen and NLR has the clinical potential as an appropriate prognostic indicator for patients suffering from cancer. F-NLR could increase the unfavorable effect of fibrinogen or NLR alone, which eventually adds to the application in predicting tumor progression. ${ }^{17}$ Yamamoto et $\mathrm{al}^{27}$ indicated that patients with a high F-NLR score might suffer a poorer prognosis than those with a relatively low F-NLR score, which is consistent with the results of our study.

In the study, we retrospectively collected the preoperative clinical data from 281 EOC patients involved. According to the ROC curve, we divided the included patients into three groups by setting a cut-off value of 
A
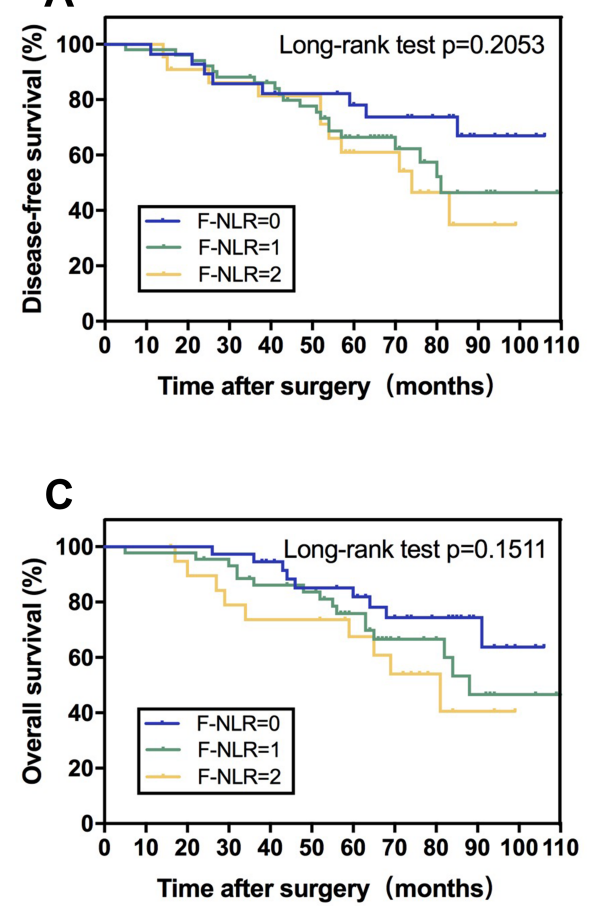
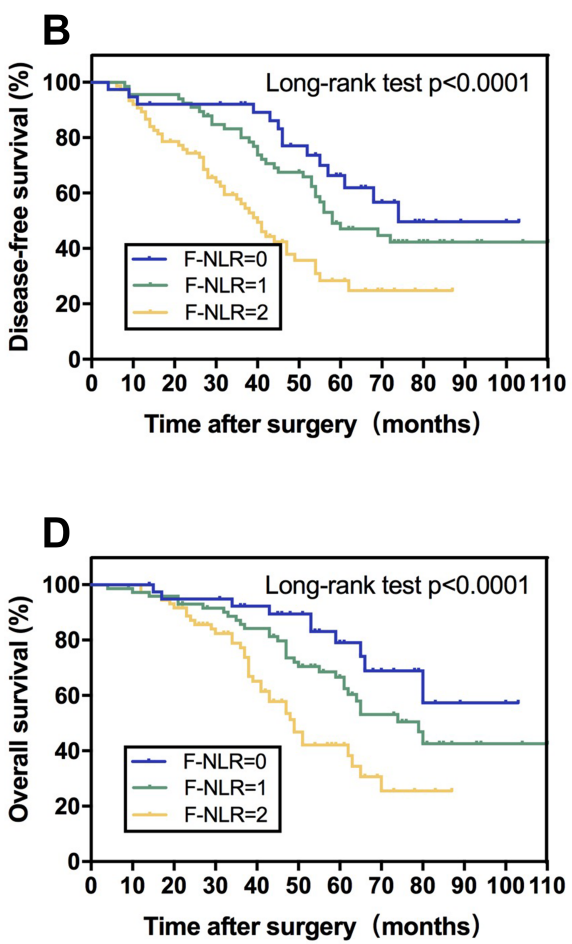

Figure 6 Kaplan-Meier survival curves stratified by fibrinogen concentrations and neutrophil lymphocyte ratio (F-NLR) score among patients with (A) lymphatic metastasis negative (-) for disease-free survival (DFS), (B) lymphatic metastasis positive (+) for DFS; (C) lymphatic metastasis negative (-) for overall survival (OS); and (D) lymphatic metastasis positive $(+)$ for OS.

fibrinogen and NLR for tumor recurrence. The F-NLR showed a greater predictive value with higher sensitivity and specificity than did fibrinogen or NLR alone. We then identified a significant association between F-NLR levels and several clinicopathological features, including FIGO clinical stage, lymphatic metastasis, and CA-125 concentration. Moreover, further univariate and multivariate analyses indicated the pre-operative F-NLR score as an independent predictive factor for overall survival among EOC patients. The study revealed that the preoperative F-NLR score could stratify patients into different risk categories of ovarian cancer recurrence. Through subgroup analysis according to the FIGO clinical stage, the DFS and OS in the patients with lower F-NLR level were higher in advanced FIGO stage (Stage III or IV). However, among patients with low FIGO stage (Stage I or II), the correlation between F-NLR and tumor prognosis was statistically insignificant. Our study also indicated that the F-NLR score was significantly related to both DFS and OS upon patients with lymph node metastasis. The results could indicate the ability of F-NLR to predict tumor growth, progression, and metastasis of EOC, especially those with advanced clinical stage and lymphatic metastasis. Therefore, our study suggested that F-NLR could present a good prognostic indicator for EOC patients, though the underlying mechanism should be further explored. This information could be used to select patients with high risk of cancer recurrence, which was important for improvement of patients' prognosis. ${ }^{28}$ Since the F-NLR score is based on conventional blood examination, it could be widespread as a practical and cost-effective indicator with promising value. $^{29}$

However, there were also some potential limitations in this study. Firstly, the present study was a retrospective analysis planned by a single institution. Next, the study enrolled a relatively small sample size of 281 EOC patients, which might be insufficient to strengthen the results. In addition, since we involved patients who underwent an optimal tumor debulking operation without preoperative treatments (such as neoadjuvant chemotherapy or radiotherapy) the analysis might have a selection bias. Accordingly, although prominent clinical values of the F-NLR score as preliminary data is shown, further multicenter prospective studies with larger sample size are still required to confirm the present hypothesis.

In conclusion, the grading system based on preoperative F-NLR score has clinical potential as a predictive marker for EOC prognosis, especially those with advanced clinical stage or lymph node metastasis. 
The F-NLR score might enable suitable risk classification of EOC patients and allow assignment of precise treatment. We hope that this economical and reliable inflammation-based index could serve as a routine biomarker for predicting prognosis upon EOC patients.

\section{Funding}

This research did not receive any specific grant from funding agencies in the public, commercial, or not-forprofit sectors.

\section{Disclosure}

The authors have declared that no competing interest exists.

\section{References}

1. Siegel RL, Miller KD, Jemal A. Cancer statistics, 2020. CA Cancer J Clin. 2020;70(1):7-30. doi:10.3322/caac.21590

2. Bray F, Ferlay J, Soerjomataram I, Siegel RL, Torre LA, Jemal A. Global cancer statistics 2018: GLOBOCAN estimates of incidence and mortality worldwide for 36 cancers in 185 countries. CA Cancer J Clin. 2018;68(6):394-424. doi:10.3322/caac.21492

3. Jacobs IJ, Menon U, Ryan A, et al. Ovarian cancer screening and mortality in the UK collaborative trial of ovarian cancer screening (UKCTOCS): a randomised controlled trial. Lancet. 2016;387 (10022):945-956. doi:10.1016/S0140-6736(15)01224-6

4. Ozols RF, Bundy BN, Greer BE, et al. Phase III trial of carboplatin and paclitaxel compared with cisplatin and paclitaxel in patients with optimally resected stage III ovarian cancer: a gynecologic oncology group study. J Clin Oncol. 2003;21(17):3194-3200. doi:10.1200/ JCO.2003.02.153

5. Bowtell DD, Bohm S, Ahmed AA, et al. Rethinking ovarian cancer II: reducing mortality from high-grade serous ovarian cancer. Nat Rev Cancer. 2015;15(11):668-679. doi:10.1038/nrc4019

6. Montagnana M, Benati M, Danese E. Circulating biomarkers in epithelial ovarian cancer diagnosis: from present to future perspective. Ann Transl Med. 2017;5(13):276. doi:10.21037/atm .2017 .05 .13

7. Polterauer S, Vergote I, Concin N, et al. Prognostic value of residual tumor size in patients with epithelial ovarian cancer FIGO stages IIA-IV: analysis of the OVCAD data. Int J Gynecol Cancer. 2012;22 (3):380-385. doi:10.1097/IGC.0b013e31823de6ae

8. Soletormos G, Duffy MJ, Othman Abu Hassan S, et al. Clinical use of cancer biomarkers in epithelial ovarian cancer: updated guidelines from the european group on tumor markers. Int $J$ Gynecol Cancer. 2016;26(1):43-51. doi:10.1097/IGC.0000000000000586

9. Hanahan D, Weinberg RA. Hallmarks of cancer: the next generation. Cell. 2011;144(5):646-674. doi:10.1016/j.cell.2011.02.013

10. McNamara MG, Templeton AJ, Maganti M, et al. Neutrophil/lymphocyte ratio as a prognostic factor in biliary tract cancer. Eur $J$ Cancer. 2014;50(9):1581-1589. doi:10.1016/j.ejca.2014.02.015

11. Cho IR, Park JC, Park CH, et al. Pre-treatment neutrophil to lymphocyte ratio as a prognostic marker to predict chemotherapeutic response and survival outcomes in metastatic advanced gastric cancer. Gastric Cancer. 2014;17(4):703-710. doi:10.1007/s10120013-0330-2
12. Mantovani A. The inflammation - cancer connection. FEBS J. 2018;285(4):638-640. doi:10.1111/febs.14395

13. McNally PW, Warriss PD. Recent bruising in cattle at abattoirs. Vet Rec. 1996;138(6):126-128. doi:10.1136/vr.138.6.126

14. Tennent GA, Brennan SO, Stangou AJ, O'Grady J, Hawkins PN, Pepys MB. Human plasma fibrinogen is synthesized in the liver. Blood. 2007;109(5):1971-1974. doi:10.1182/blood-2006-08-040956

15. Ghezzi F, Cromi A, Siesto G, et al. Prognostic significance of preoperative plasma fibrinogen in endometrial cancer. Gynecol Oncol. 2010;119(2):309-313. doi:10.1016/j.ygyno.2010.07.014

16. Arigami T, Uenosono Y, Matsushita D, et al. Combined fibrinogen concentration and neutrophil-lymphocyte ratio as a prognostic marker of gastric cancer. Oncol Lett. 2016;11(2):1537-1544. doi:10.3892/ ol.2015.4049

17. Wang H, Zhao J, Zhang M, Han L, Wang M, Xingde L. The combination of plasma fibrinogen and neutrophil lymphocyte ratio (F-NLR) is a predictive factor in patients with resectable non small cell lung cancer. $J$ Cell Physiol. 2018;233(5):4216-4224. doi:10. $1002 /$ jcp. 26239

18. Palumbo JS, Talmage KE, Massari JV, et al. Platelets and fibrin(ogen) increase metastatic potential by impeding natural killer cell-mediated elimination of tumor cells. Blood. 2005;105(1):178-185. doi:10. 1182/blood-2004-06-2272

19. Son HJ, Park JW, Chang HJ, et al. Preoperative plasma hyperfibrinogenemia is predictive of poor prognosis in patients with nonmetastatic colon cancer. Ann Surg Oncol. 2013;20(9):2908-2913. doi:10.1245/s10434-013-2968-8

20. Chiarug V, Ruggiero M, Magnelli L. Angiogenesis and the unique nature of tumor matrix. Mol Biotechnol. 2002;21(1):85-90. doi:10.1385/MB:21:1:085

21. Sahni A, Simpson-Haidaris PJ, Sahni SK, Vaday GG, Francis CW. Fibrinogen synthesized by cancer cells augments the proliferative effect of fibroblast growth factor-2 (FGF-2). J Thromb Haemost. 2008;6(1):176-183. doi:10.1111/j.1538-7836.2007.02808.x

22. Mantovani A, Allavena P, Sica A, Balkwill F. Cancer-related inflammation. Nature. 2008;454(7203):436-444. doi:10.1038/nature 07205

23. Cools-Lartigue J, Spicer J, McDonald B, et al. Neutrophil extracellular traps sequester circulating tumor cells and promote metastasis. J Clin Invest. 2013;123(8):3446-3458. doi:10.1172/JCI67484

24. Dworacki G, Meidenbauer N, Kuss I, et al. Decreased zeta chain expression and apoptosis in CD3+ peripheral blood $\mathrm{T}$ lymphocytes of patients with melanoma. Clin Cancer Res. 2001;7(3Suppl):947s-957s.

25. Kusumanto YH, Dam WA, Hospers GA, Meijer C, Mulder NH. Platelets and granulocytes, in particular the neutrophils, form important compartments for circulating vascular endothelial growth factor. Angiogenesis. 2003;6(4):283-287. doi:10.1023/B:AGEN.0000029415.62384.ba

26. Donskov F. Immunomonitoring and prognostic relevance of neutrophils in clinical trials. Semin Cancer Biol. 2013;23(3):200-207. doi:10.1016/j.semcancer.2013.02.001

27. Yamamoto M, Kurokawa Y, Kobayashi N, et al. Prognostic value of the combined index of plasma fibrinogen and the neutrophil-lymphocyte ratio in gastric cancer. World J Surg. 2020;44(1):207-212. doi:10.1007/ s00268-019-05193-7

28. Kijima T, Arigami T, Uchikado Y, et al. Combined fibrinogen and neutrophil-lymphocyte ratio as a prognostic marker of advanced esophageal squamous cell carcinoma. Cancer Sci. 2017;108 (2):193-199. doi:10.1111/cas.13127

29. Zhu Y, Zhou S, Liu Y, Zhai L, Sun X. Prognostic value of systemic inflammatory markers in ovarian cancer: a PRISMA-compliant meta-analysis and systematic review. BMC Cancer. 2018;18(1):443. doi:10.1186/s12885-018-4318-5 


\section{Publish your work in this journal}

OncoTargets and Therapy is an international, peer-reviewed, open access journal focusing on the pathological basis of all cancers, potential targets for therapy and treatment protocols employed to improve the management of cancer patients. The journal also focuses on the impact of management programs and new therapeutic

Submit your manuscript here: https://www.dovepress.com/oncotargets-and-therapy-journal agents and protocols on patient perspectives such as quality of life, adherence and satisfaction. The manuscript management system is completely online and includes a very quick and fair peer-review system, which is all easy to use. Visit http://www.dovepress.com/ testimonials.php to read real quotes from published authors. 